\title{
Effects of flow distributor geometry and diffusion layer porosity on reactant gas transport and performance of proton exchange membrane fuel cells
}

\author{
W.M. Yan ${ }^{\text {a }}$, C.Y. Soong ${ }^{b}, *$ Falin Chen ${ }^{\text {c }}$, H.S. Chu ${ }^{\mathrm{d}}$ \\ ${ }^{a}$ Department of Mechatronic Engineering, HuaFan University, Shih-Ting, Taipei 223, Taiwan, ROC \\ b Department of Aeronautical Engineering, Feng Chia University, 100 Wenhua Road, Seatwen, Taichung 407, Taiwan, ROC \\ ${ }^{\mathrm{c}}$ Institute of Applied Mechanics, National Taiwan University, Taipei 107, Taiwan, ROC \\ ${ }^{\mathrm{d}}$ Department of Mechanical Engineering, National Chiao Tung University, Hsinchu 300, Taiwan, ROC
}

Received 22 May 2003; accepted 21 July 2003

\begin{abstract}
A study has been conducted on the effects of flow distributor and diffusion layer geometries and morphologies on the transport phenomena of reactant gas from flow channels to gas diffuser, and on the performance of a proton exchange membrane fuel cell. The emphasis of the analysis is placed on the effects of the cross-section of the channel of the anode flow distributor and the porosity of the gas-diffusion layer. By applying a two-dimensional mass transport model, the effects of the channel width fraction, $\lambda=\ell_{\mathrm{c}} / \ell_{\mathrm{b}}$, the channel number, $N$, the porosity of the diffuser layer, $\varepsilon$, and the surface overpotential of the catalyst layer, $\eta$, on the resultant current density are investigated. The cell performance is evaluated by the predicted voltage-current density curves. It is disclosed that an increase in either $\lambda, N$ or $\varepsilon$ may lead to a better cell performance. At relatively low overpotential, better uniformity in current density distribution along the width of the cell ( $x$-direction) can be attained. Based on the present results, a correlation of overpotentials is proposed, namely, $\eta_{\mathrm{cr}}=0.165 \lambda^{0.0935} N^{0.2033} \varepsilon^{0.0856}$, which may serve as a guideline to justify the validity of a simplified one-dimensional model.
\end{abstract}

(C) 2003 Elsevier B.V. All rights reserved.

Keywords: Proton exchange fuel cell; Flow channel design; Gas-diffusion layer; Overpotential; Porosity

\section{Introduction}

The proton exchange membrane fuel cell (PEMFC) is a most promising energy source due to its environmental attributes as well as its high efficiency The latter feature depends strongly on the operating conditions, transport phenomena in the cells, electrochemical reaction kinetics, mechanical design and manufacturing process, etc. Crucial among these is the reactant gas transport in the flow distributor and the gas-diffusion layer before the reactant gas reaches the catalyst layer to carry out reaction. Different types of flow channel, such as the serpentine channel, multiple channels in parallel type and interdigited channels, have been analyzed in order to reach optimization of the cell performance. The geometric parameters and the morphologies of the gas-diffusion layer and the catalyst layer have also been investigated.

\footnotetext{
* Corresponding author. Fax: +886-4-24516246.

E-mail address: cysoong@fcu.edu.tw (C.Y. Soong).
}

West and Fuller [1] studied the effects of the rib spacing of bipolar plates and the thickness of the gas-diffusion electrode on cell performance. It was found that the two-dimensional effect only slightly alters the half-cell potential for a given applied current, but has a significant influence on water management. Jordan et al. [2] examined the influence of diffusion-layer morphology on cell performance. A model of the hydrophobilicity and the porosity of the gas-diffusion layer was used to explain the influence of the diffusion-layer morphology. In other work [3], these authors obtained results which showed that a low-porosity acetylene black enhances water removal from the membrane electrode assembly (MEA), and thus gives an improvement in gas-diffusion. Guran et al. [4] proposed a mathematical model to obtain an analytic solution of the mass transport of reactant gas in a half-cell, in which the effects of the porosity and the tortuosity of the gas-diffusion layer and the catalyst layer were explored.

To obtain details of the flow distribution and gas-diffusion, numerical computations have been carried out recently by a few investigators. For example, Dutta et al. [5] solved 


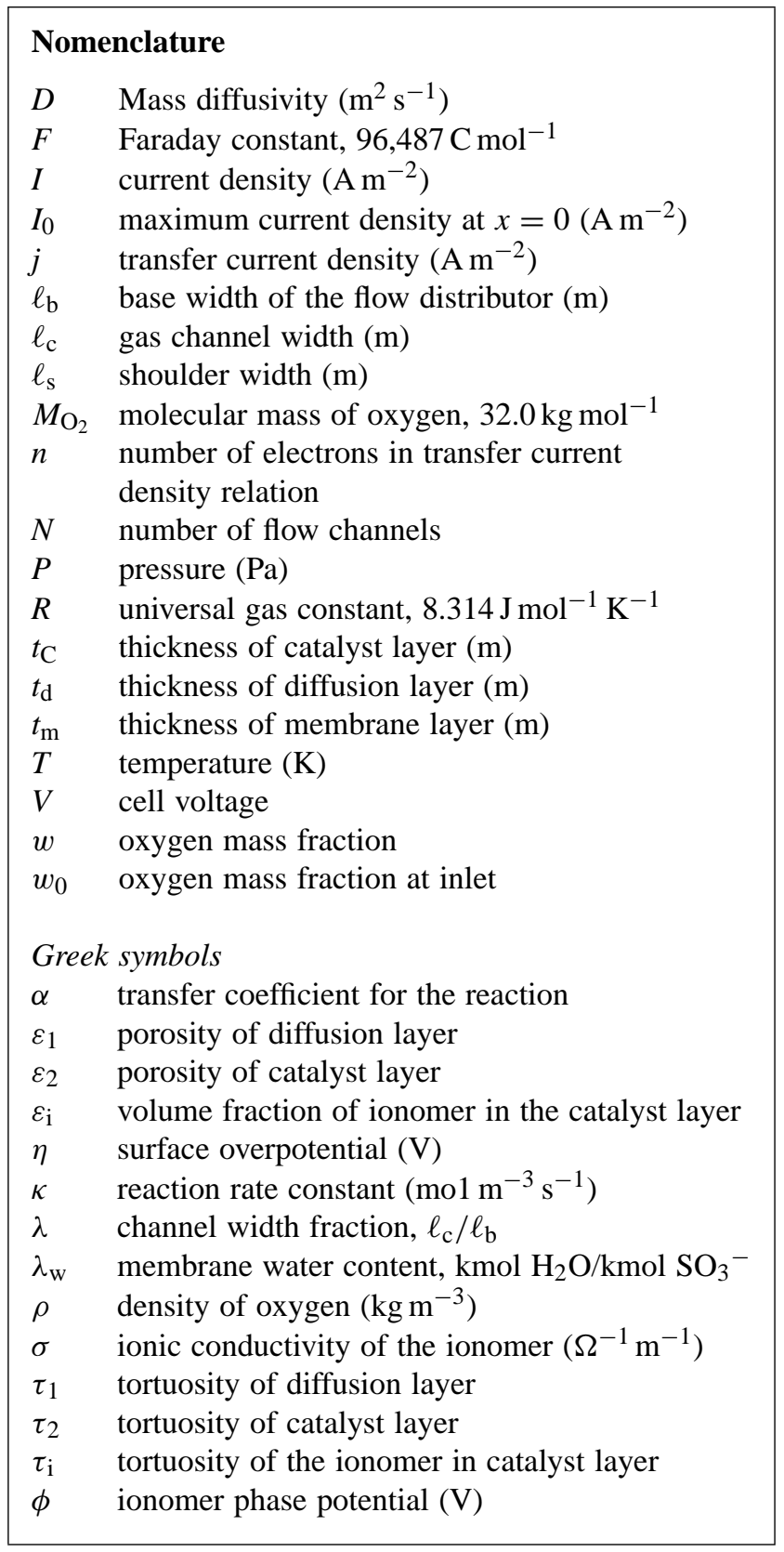

three-dimensional Navier-Stokes equations with a electrochemical relation model for the mass source/sink terms to examine the mass exchange in serpentine flow channels at both the cathode and the anode. To achieve optimization of the flow field and to enhance fuel cell performance, Hontanon et al. [6] focused attention on the flow distributor rather than the whole cell. Two types of flow distributors, were evaluated, namely, grooved plate and porous material, by employing Navier-Stokes equations as the flow model and Michaelis-Menten type two-step kinetics as the gas reaction model at the anode. The computational results showed that fuel consumption can be increased by decreasing the permeability of the flow distributor, especially when the permeability decreases to a value below that of the anode. In terms of the reactant gas utilization based on the same pressure drop, it was claimed that porous materials are more advantageous than grooved plates. For flow distribution in the channel networks of typical planar fuel cell layers and stacks, Kee et al. [7] have recently proposed a generalized computational model, in which only mass and momentum transfer are considered.

The objective of the present work is to study the effects of the flow distributor and the diffusion layer morphologies on the transport of reactant gas from the flow channels to the gas diffuser, and on the performance of a PEMFC. Emphasis is placed on the effects of the cross-section of the flow in terms of the ratio of the channel width $\ell_{\mathrm{c}}$ to the base plate width $\ell_{\mathrm{b}}$, i.e. $\lambda=\ell_{\mathrm{c}} / \ell_{\mathrm{b}}$, and the morphology of the gas-diffusion layer in terms of the porosity of the layer. By applying a mass transport model, the effects of these two parameters and the overpotential on the resultant current density are investigated and the cell performance is evaluated by the voltage-current density or performance curves. Based on the results with various overpotentials, a correlation is proposed to serve as a guideline for justification of the validity of a simplified one-dimensional model.

\section{Theory}

\subsection{Model development}

A schematic diagram of the cross-sectional view of a half-PEMFC is shown in Fig. 1. The shoulder area, which is essential for electronic transport from the electrode to the current-collector, affects the fuel cell performance by changing the ohmic and contact resistance in the cell. Therefore,
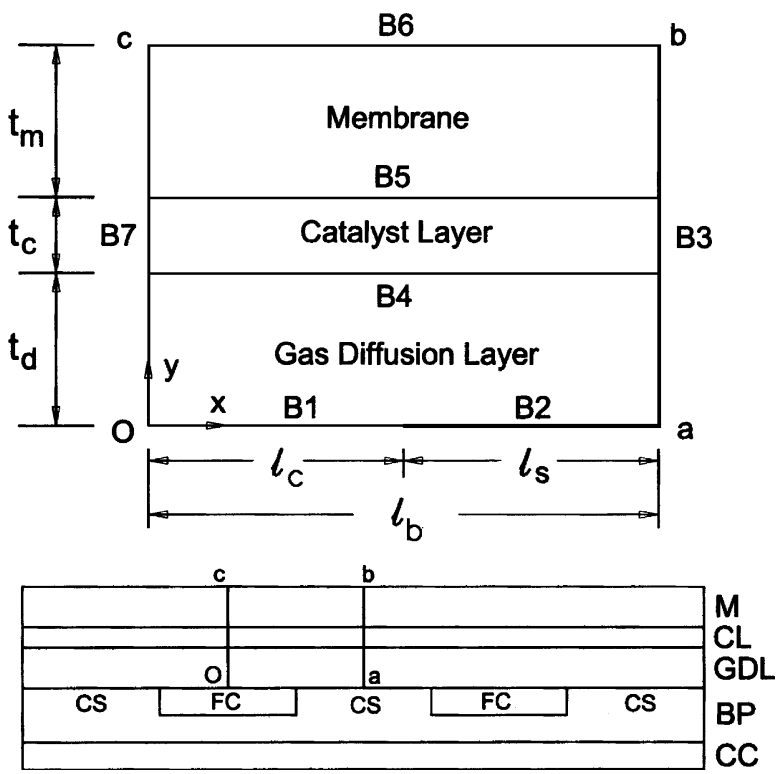

Fig. 1. Half-cell model of a PEMFC. FC: flow channel; CS: channel shoulder; CC: current collector; BP: bipolar plate; GDL: gas-diffusion layer; CL: catalyst layer; M: membrane; B1-B7: boundary numbers. 
it is important to investigate the effects of shoulder area on the transport phenomena of the reactant through the cathode and on the ohmic resistance of the flow distributor. Moreover, the area ratio between flow channel and shoulder needs to be optimized for both gas transports and electronic requirements.

Oxygen gas transport through a half-PEMFC is simulated. Gas transport from the flow channels to the catalyst is by diffusion, which is driven by the concentration gradient. A steady-state, two-dimensional transport equation for the oxygen mass fraction, $w$, in the diffusion layer can be expressed by:

$\frac{\partial}{\partial x}\left(\varepsilon_{1}^{\tau_{1}} \rho D \frac{\partial w}{\partial x}\right)+\frac{\partial}{\partial y}\left(\varepsilon_{1}^{\tau_{1}} \rho D \frac{\partial w}{\partial x}\right)=0$

where $\rho$ is the gas density; $D$ the mass diffusivity; $\varepsilon_{1}$ and $\tau_{1}$ are the porosity and tortuosity factor of the diffusion catalyst layer, respectively. In the catalyst layer, the oxygen is consumed and the transport equation becomes:

$\frac{\partial}{\partial x}\left(\varepsilon_{2}^{\tau_{2}} \rho D \frac{\partial w}{\partial x}\right)+\frac{\partial}{\partial y}\left(\varepsilon_{2}^{\tau_{2}} \rho D \frac{\partial w}{\partial y}\right)-\frac{j}{2 n F} M_{\mathrm{O}_{2}}=0$

where $j$ is the transfer current density; $n$ the number of electrons in the transfer current density relation; $F$ the Faraday constant; $\varepsilon_{2}$ and $\tau_{2}$ are the porosity and the tortuosity factor of the catalyst layer, respectively.

Assuming that the oxygen reduction reaction (ORR) is irreversible and first order in oxygen, and that electroneutrality holds in any representative elementary volume, the cathode transfer current density can be expressed as:

$j=n F \kappa w \exp \left(\frac{\alpha n F}{R T}\right)$.

Substituting Eq. (4) into (2), gives:

$$
\begin{gathered}
\frac{\partial}{\partial x}\left(\varepsilon_{2}^{\tau_{2}} \rho D \frac{\partial w}{\partial x}\right)+\frac{\partial}{\partial y}\left(\varepsilon_{2}^{\tau_{2}} \rho D \frac{\partial w}{\partial y}\right) \\
-\frac{\kappa M_{\mathrm{O}_{2}}}{2} \exp \left(\frac{\alpha n F}{R T} \eta\right) w=0 .
\end{gathered}
$$

The expression for the membrane phase potential is:

$$
\frac{\partial}{\partial x}\left(\sigma \frac{\partial \phi}{\partial x}\right)+\frac{\partial}{\partial y}\left(\sigma \frac{\partial \phi}{\partial y}\right)=0 .
$$

In the catalyst layer with the presence of transfer current density, the phase potential equation is:

$\frac{\partial}{\partial x}\left(\varepsilon_{\mathrm{i}}^{\tau_{\mathrm{i}}} \sigma \frac{\partial \phi}{\partial x}\right)+\frac{\partial}{\partial y}\left(\varepsilon_{\mathrm{i}}^{\tau_{\mathrm{i}}} \sigma \frac{\partial \phi}{\partial y}\right)=j$,

where $\varepsilon_{\mathrm{i}}$ and $\tau_{\mathrm{i}}$ are the volume fraction and the tortuosity factor of the ionomer in the catalyst layer; $\lambda_{\mathrm{w}}$ the membrane water content; $\sigma$ is the membrane ionic conductivity and is evaluated by:

$$
\begin{aligned}
\sigma= & \left(0.5139 \lambda_{\mathrm{w}}-0.326\right) \exp \\
& \times\left[1268\left(\frac{1}{303}-\frac{1}{T}\right) \times 10^{-2}\right] .
\end{aligned}
$$

\subsection{Boundary conditions}

The conditions subjected to the boundaries numbered B1-B7 in Fig. 1 are described as follows. The boundary conditions for the oxygen mass fraction, $w$, are:

$w=w_{0}, \quad$ at boundary $\mathrm{B} 1$

$\frac{\partial w}{\partial y}=0, \quad$ at boundaries B2 and B5

$\frac{\partial w}{\partial y}=0, \quad$ at boundaries B3 and B7

$w_{-}=w_{+}, \quad \varepsilon_{1}^{\tau_{1}} D \frac{\partial w}{\partial y}=\varepsilon_{2}^{\tau_{2}} D \frac{\partial w}{\partial y}, \quad$ at boundary B4.

The boundary conditions for potential $\phi$ are:

$\frac{\partial \phi}{\partial y}=0, \quad$ at boundary B4

$\frac{\partial \phi}{\partial y}=0, \quad$ at boundaries B3 and B7

$\phi_{-}=\phi_{+},\left.\quad \varepsilon_{3}^{\tau_{3}} \frac{\partial \phi}{\partial y}\right|_{-}=\left.\frac{\partial \phi}{\partial y}\right|_{+}$, at boundary B5

$\phi=0, \quad$ at boundary B6.

\subsection{Evaluation of cell potential}

With the potentials given by the above model, the cell potential $V$ can be calculated by the following equation:

$V=V_{\mathrm{oc}}-\eta-\Delta \phi$

where $V_{\mathrm{oc}}$ is the ideal cell potential; $\eta$ the surface overpotential; $\Delta \phi$ is the average phase potential difference and can be expressed as:

$\Delta \phi=\int_{0}^{\ell_{\mathrm{b}}}\left(\phi_{6}-\phi_{4}\right) \frac{\mathrm{d} x}{\ell_{\mathrm{b}}}$

in which $\ell_{\mathrm{b}}=\ell_{\mathrm{c}}+\ell_{\mathrm{s}}$ is the base width (one-half of a channel-shoulder pitch), and $\phi_{4}$ and $\phi_{6}$ denote phase potentials at boundaries B4 and B6, respectively.

\section{Results and discussion}

The model described above is solved by a finite difference method with a uniformly distributed grid having 201 and 221 points in the $x$ and $y$ directions, respectively. The values of the parameters considered in the present study are listed in Table 1. The numerical results are presented to discuss the effects of some physical and morphological parameters. The geometry is characterized by a parameter named the channel width fraction, $\lambda \equiv \ell_{\mathrm{c}} / \ell_{\mathrm{b}}$, of which three values, $\lambda=0.25,0.5$, and 0.75 are considered to explore the 
Table 1

Parameters used in present analysis

\begin{tabular}{|c|c|c|}
\hline Parameters & Notation & Value \\
\hline Temperature (K) & $T$ & 333 \\
\hline Pressure $(\mathrm{Pa})$ & $P$ & 303975 \\
\hline Reaction rate constant $\left(\mathrm{mol} \mathrm{m}^{-3} \mathrm{~s}^{-1}\right)$ & $\kappa$ & $1.5 \times 10^{-5}$ \\
\hline Oxygen mass fraction at inlet & $w_{0}$ & 0.17 \\
\hline Gas channel width (m) & $\ell_{\mathrm{c}}$ & $\begin{array}{l}\text { (a) 4-channel, } 5 \times 10^{-4} \\
\text { (b) } 2 \text {-channel, } 1.0 \times 10^{-3} \\
\text { (c) Single-channel, } 2.0 \times 10^{-3}\end{array}$ \\
\hline Channel shoulder width (m) & $\ell_{\mathrm{s}}$ & $\begin{array}{l}\text { (d) 4-channel, } 5 \times 10^{-4} \\
\text { (e) } 2 \text {-channel, } 1.0 \times 10^{-3} \\
\text { (f) Single-channel, } 2.0 \times 10^{-3}\end{array}$ \\
\hline Thickness of diffusion layer (m) & $t_{\mathrm{d}}$ & $5 \times 10^{-4}$ \\
\hline Thickness of catalyst layer (m) & $t_{\mathrm{C}}$ & $5 \times 10^{-5}$ \\
\hline Thickness of membrane $(\mathrm{m})$ & $t_{\mathrm{m}}$ & $2.0 \times 10^{-4}$ \\
\hline Porosity of GDL & $\varepsilon_{1}$ & 0.8 \\
\hline Tortuosity of GDL & $\tau_{1}$ & 1.5 \\
\hline Porosity of catalyst layer & $\varepsilon_{2}$ & 0.3 \\
\hline Tortuosity of catalyst layer & $\tau_{2}$ & 1.5 \\
\hline Volume fraction of ionomer in $\mathrm{CL}$ & $\varepsilon_{3}$ & 0.3 \\
\hline Ionomer tortuosity in $\mathrm{CL}$ & $\tau_{3}$ & 1.5 \\
\hline Faraday constant $\left(\mathrm{C} \mathrm{mol}^{-1}\right)$ & $F$ & 96487 \\
\hline Universal gas constant $\left(\mathrm{kJ} \mathrm{kmol}^{-1} \mathrm{~K}^{-1}, \mathrm{~J} \mathrm{~mol}^{-1} \mathrm{~K}^{-1}\right)$ & $R$ & 8.314 \\
\hline Molecular mass of oxygen $\left(\mathrm{kg} \mathrm{kmol}^{-1}\right)$ & $M$ & 32.0 \\
\hline Number of electrons in relation participating & $n$ & 2 \\
\hline Current density $\left(\mathrm{A} \mathrm{m}^{-2}\right)$ & $I$ & \\
\hline Surface overpotential (V) & $\eta$ & \\
\hline Ionomer phase potential $(\mathrm{V})$ & $\phi$ & \\
\hline
\end{tabular}

geometric effects of the flow channel on the performance. In addition, the number of flow channels, $N$, is also an important parameter worthy of investigation. For a fixed channel width fraction, $\lambda=0.5$, three configurations of $N=1$, 2 , and 4 are studied. Finally, to explore the effects of the porosity of the gas-diffusion layer, various values of porosity are considered. Without loss of generality, the porosities of the catalyst and membrane have the same value as that of the gas-diffusion layer, i.e. $\varepsilon_{2}=\varepsilon_{i}=\varepsilon_{1}$, and porosity in the range of $0.3 \leq \varepsilon_{1} \leq 0.6$ are examined. In the following discussion, unless otherwise stated, the results are generated at the condition $\varepsilon_{1}=0.3$.

\subsection{Effect of channel width fraction}

The contours of the oxygen mass fraction in the diffusion layer $\left(0 \leq y \leq 5 \times 10^{-4}\right)$ and the catalyst layer $\left(5 \times 10^{-4} \leq\right.$ $y \leq 5.5 \times 10^{-4}$ ) of $\varepsilon_{1}=0.3$ in the case of $N=2, \eta=0.2$ and $\lambda=0.25,0.5$ and 0.75 are presented in Fig. 2 . The bold line on the bottom right of each contour marks the axial location of the shoulder (or rib) of the channel. In the region corresponding to the channel central region (left portion of the contour), a relatively higher oxygen mass fraction appears and, then, the oxygen diffuses towards the porous material that covers the rib where there is no oxygen source on the interface $y=0$. The concentration gradient of the oxygen is more noticeable in the case of a smaller channel width fraction, i.e. $\lambda=0.25$, and with increasing channel width, the gradient becomes moderate. The phase potential distributions in the catalyst layer $\left(5 \times 10^{-4} \leq y \leq 5.5 \times 10^{-4}\right)$ and the membrane $\left(5.5 \times 10^{-4} \leq y \leq 7.5 \times 10^{-4}\right)$ of porosity $\varepsilon=0.3$ are presented in Fig. 3. It is found that an increase in the channel width fraction alleviates the potential gradient in the $x$-direction. Additionally, at a large value of $\lambda$, the total difference between the potentials $\phi_{6}$ at $y=7.5 \times 10^{-4}$ and $\phi_{4}$ at $y=5 \times 10^{-4}$ along the $x$-direction, $\Delta \phi=$ $\int_{0}^{\ell_{\mathrm{b}}}\left(\phi_{6}-\phi_{4}\right) \mathrm{d} x / \ell_{\mathrm{b}}$, becomes small and, from Eq. (10), the cell potential increases. This phenomenon is attributed to a more uniform distribution of oxygen (Fig. 3).

The current density distribution at the interface between bipolar plate and gas-diffusion layer, i.e. boundaries B1 and B2 in Fig. 1, under various given conditions of surface overpotential, $\eta$, is shown in Fig. 4. With periodic conditions at left and right sides, i.e. boundaries B3 and B7, the effects of relative channel width are explored by examining the current density distributions in the $x$-direction. It is observed that the distribution of the current density in the $x$-direction at the interface of the gas-diffusion layer and the catalyst layer can be uniform at low overpotentials. At a high overpotential, the surface reaction rate becomes fast and the relatively weak diffusion leads to a non-uniform current density. This situation is prevailing over the various channel width fractions considered, i.e. $\lambda=0.25,0.5$, and 0.75 .

Current density distribution with variation of overpotential is plotted in Fig. 5. The data clearly show the level 

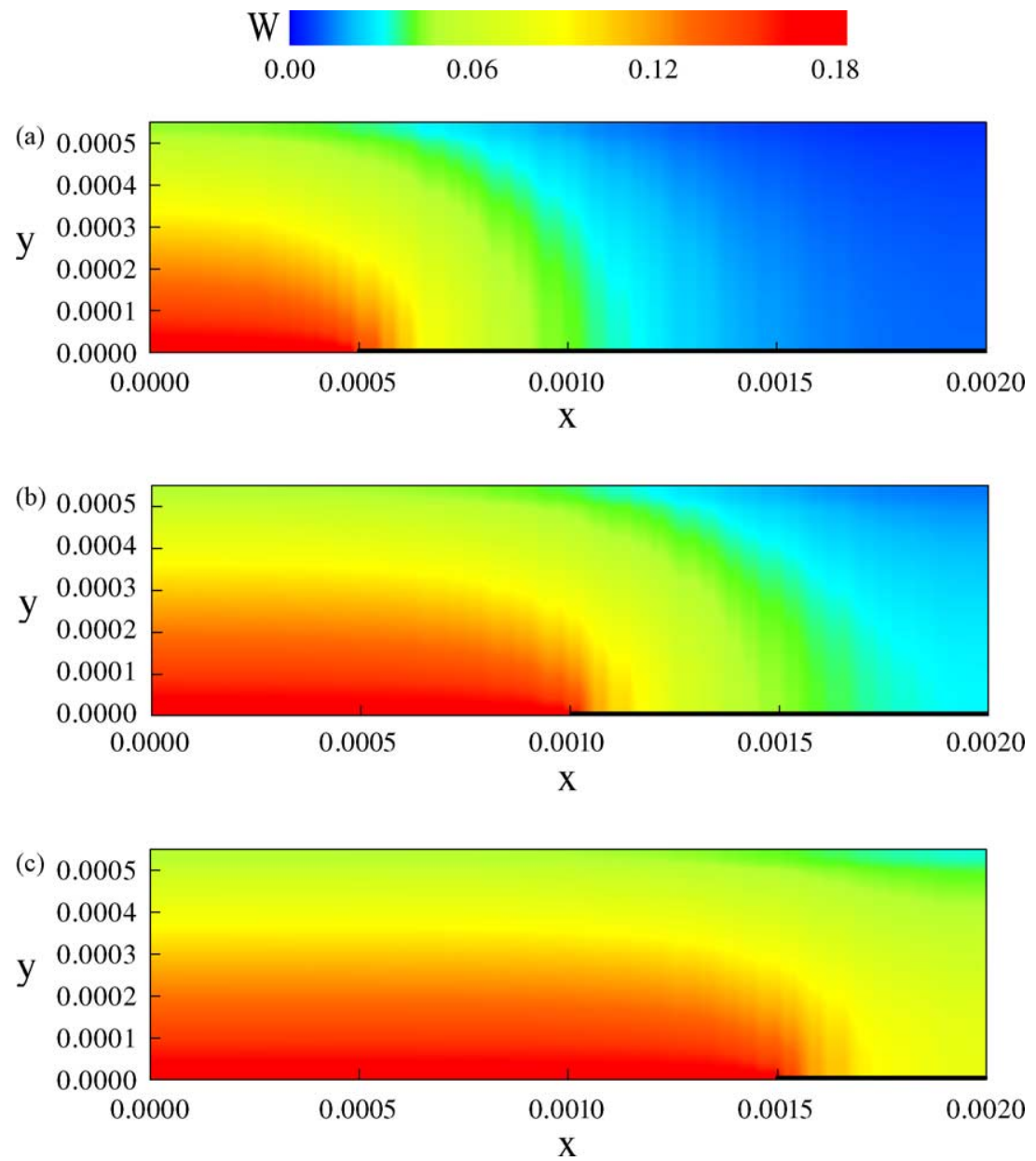

Fig. 2. Oxygen mass fraction contours in diffusion and catalyst layer with $N=2, \eta=0.25$, and (a) $\lambda=0.25$; (b) $\lambda=0.5$; (c) $\lambda=0.75$.
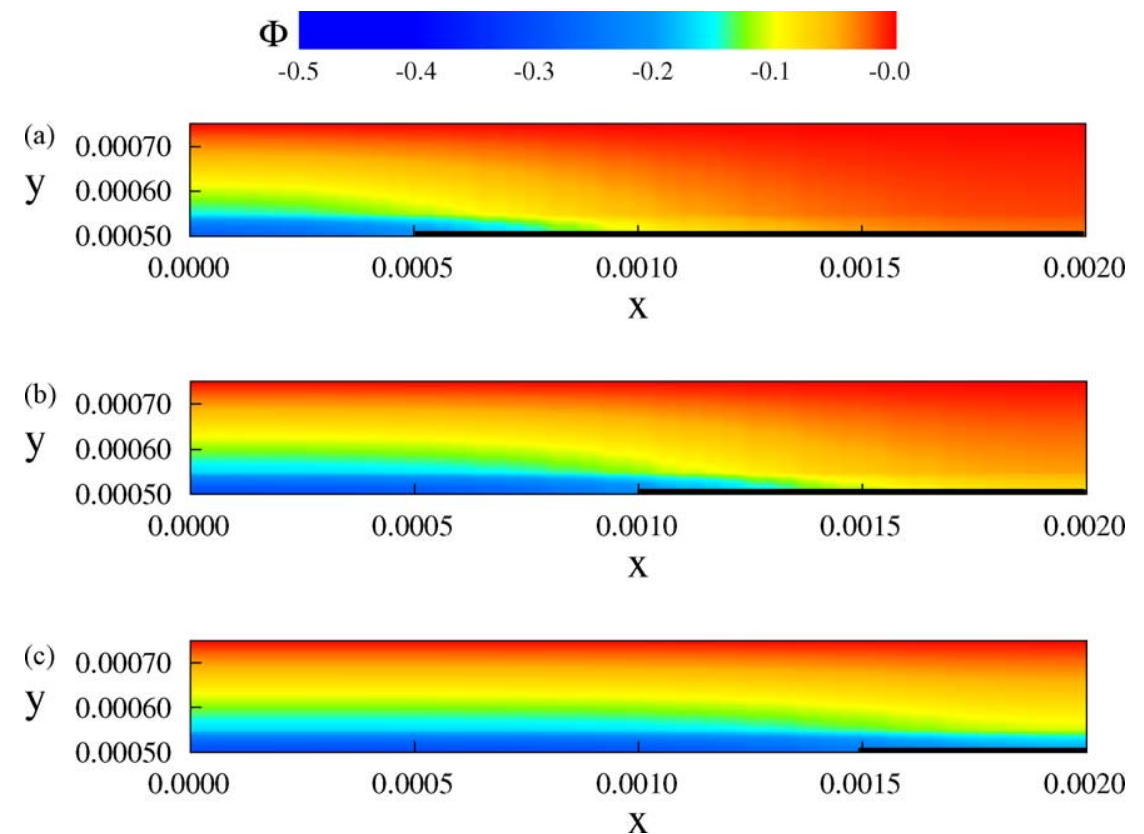

Fig. 3. Ionomer phase potential contours in catalyst layer and membrane with $N=2, \eta=0.25$, and (a) $\lambda=0.25$; (b) $\lambda=0.5$; (c) $\lambda=0.75$. 

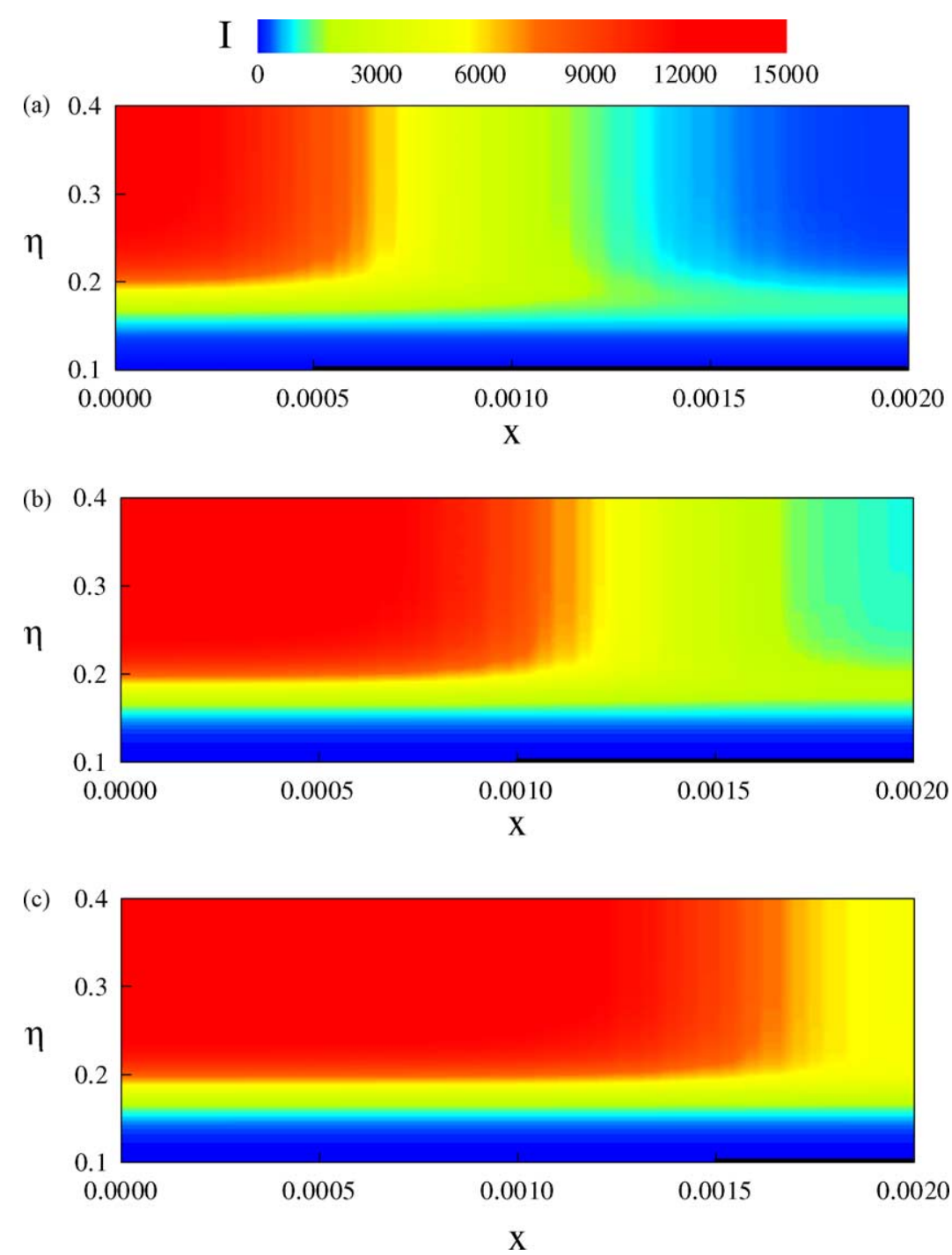

Fig. 4. Effects of surface overpotential on current density distribution along $x$-direction with $N=2$ and various channel width fractions. (a) $\lambda=0.25$; (b) $\lambda=0.5$; (c) $\lambda=0.75$.

and the non-uniformity of $I$ distribution with increasing overpotential. The maximum current density which appears at the location $x=0$ is denoted by $I_{0}$. The $\eta$-dependence of $I_{0}$ is presented in Fig. 6. It is found that, as overpotential is increased to enhance the surface reaction, the maximum current density changes dramatically. Increase in the overpotential enhances the surface reaction. The local maximum current density $I_{0}$ is a parameter can be used to characterize the level of the current density in the fuel cell model. The data in Fig. 6 disclose that the variation of $I_{0}$ can be divided into three regimes:

(i) slow reaction regime $(\eta<0.12)$-in which the current density is low and the increase in $\eta$ has little effect on $I_{0}$;

(ii) high sensitivity regime $(0.12<\eta<0.3)$-the regime is characterized by remarkable changes in $I_{0}$ with increasing $\eta$; (iii) saturation regime $(\eta>0.3)$ —where $I_{0}$ approaches an asymptotic value and further increase in $\eta$ has little effect on $I_{0}$.

The correlations of current density with overpotential in Fig. 6 demonstrate that the influences of the channel width fraction on $I_{0}$ are just moderate. The $I_{0}$ versus $\eta$ curve at $\lambda=0.5$ almost coincides with that at $\lambda=0.75$. This implies that the effect of $\lambda$ on $I_{0}$ vanishes at $\lambda \geq 0.5$. Although the maximum values of current density for the two geometries are extremely close, the distributions shown in Fig. 4(b) and (c) provide evidence of better performance with $\lambda=0.75$ than with $\lambda=0.5$. This is attributed to the higher local values of current density and the better uniformity attained in a case of larger channel width fraction. To explore the influence of channel width on performance, the $I$ versus $V$ correlations at $\lambda=0.25,0.5,0.75$ and 1.00 are presented in Fig. 7. These curves demonstrate 

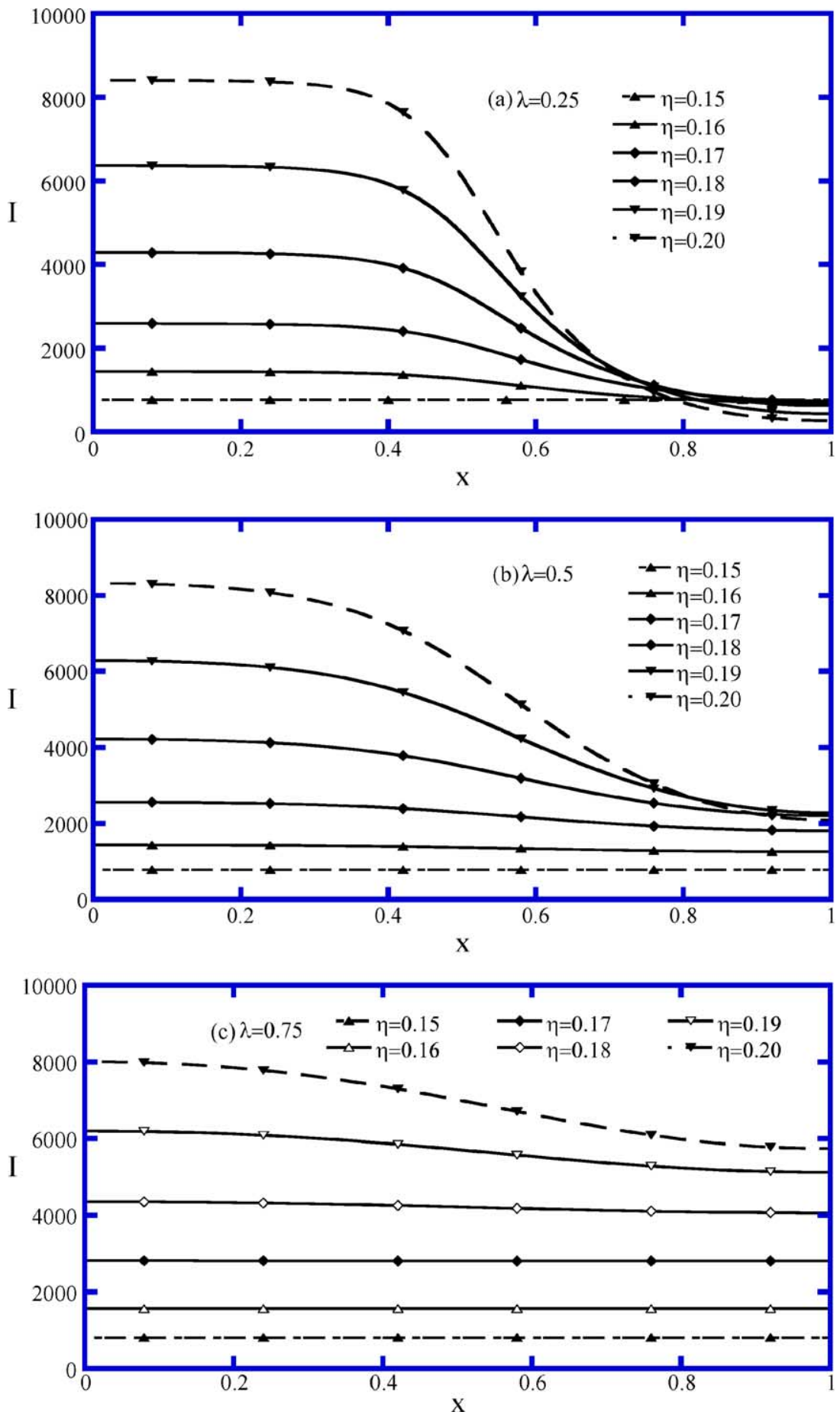

Fig. 5. Quantitative representation of current density distributions along $x$-direction corresponding to cases in Fig. 4. (a) $\lambda=0.25$; (b) $\lambda=0.5$; (c) $\lambda=0.75$.

that the larger is the channel width fraction, the better is the performance. Without consideration of the details of the electrochemical reaction and other factors, the results are reasonable from a geometric viewpoint.

\subsection{Effect of channel number}

To explore the influence of the flow channel number on the diffusion of gas reactants and the performance of the fuel cell, the models with a fixed channel width fraction, $\lambda=0.5$, are used. The effect of overpotential on the current density distribution with channel number $N=1,2$ and 4 is shown in Fig. 8, from which the effect of channel number can also be extracted. From a comparison of the local distribution of the current density at a fixed overpotential, it is found that better uniformity can be obtained for a flow channel design with a larger channel number. The inherent distributive nature of the multi-channel design is the major reason. Three-regime 


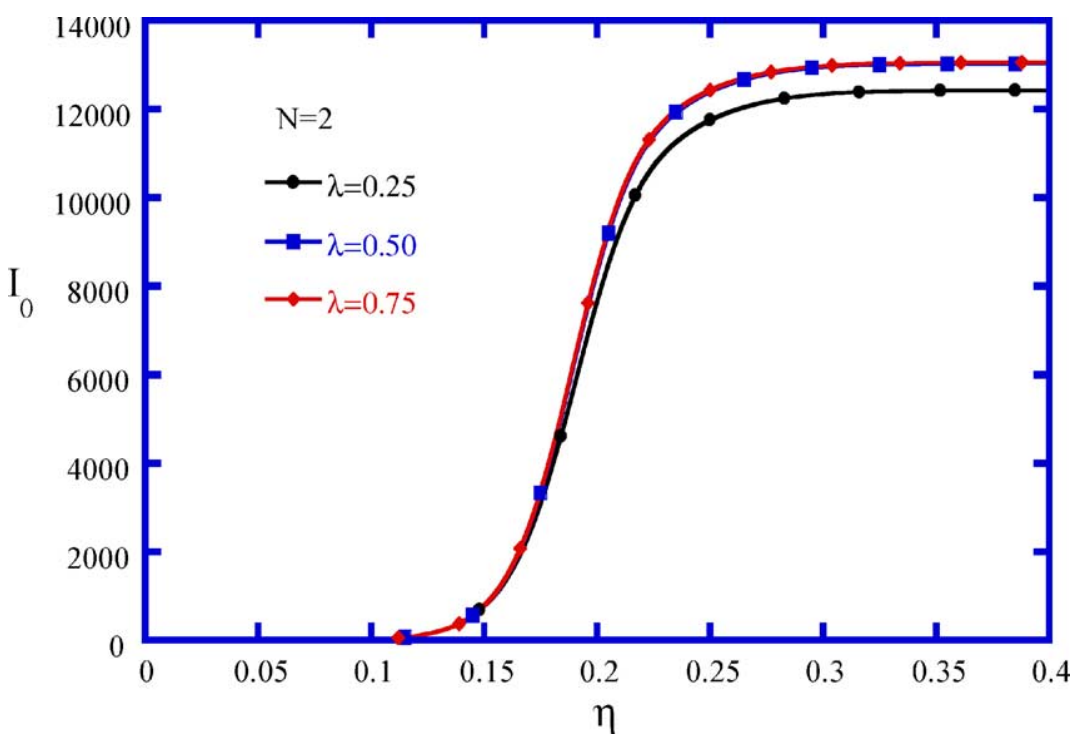

Fig. 6. Correlation of local maximum current density and surface overpotential with various channel width fractions.

nature of the maximum current density curves, i.e. $I_{0}$ versus $\eta$ plots, defined above is clearly seen in Fig. 9. The effects of channel number on the value of $I_{0}$, like that of the channel width fraction, have little significance.

The influence of the channel number on the performance, i.e. the $I$ to $V$ correlations in the cases of $N=1,2$, and 4 are presented in Fig. 10. The predictions of the performance curves reveal that a larger number of flow-channel leads to better performance for the effective distribution and utilization of reacting gas.

\subsection{Effect of gas-diffusion layer porosity}

The effect of gas-diffusion layer porosity in the range $0.3 \leq \varepsilon \leq 0.6$ on current density distribution at the interface between this layer and the catalyst layer along $x$-direction of the models with $N=2$, and $\lambda=0.5$ is shown in Fig. 11 . An increase in porosity enhances the diffusion transport of the reactant gas through the porous layers and, in turn, increases the local values of the current density with a specified overpotential. This strong transport rate of the reactant

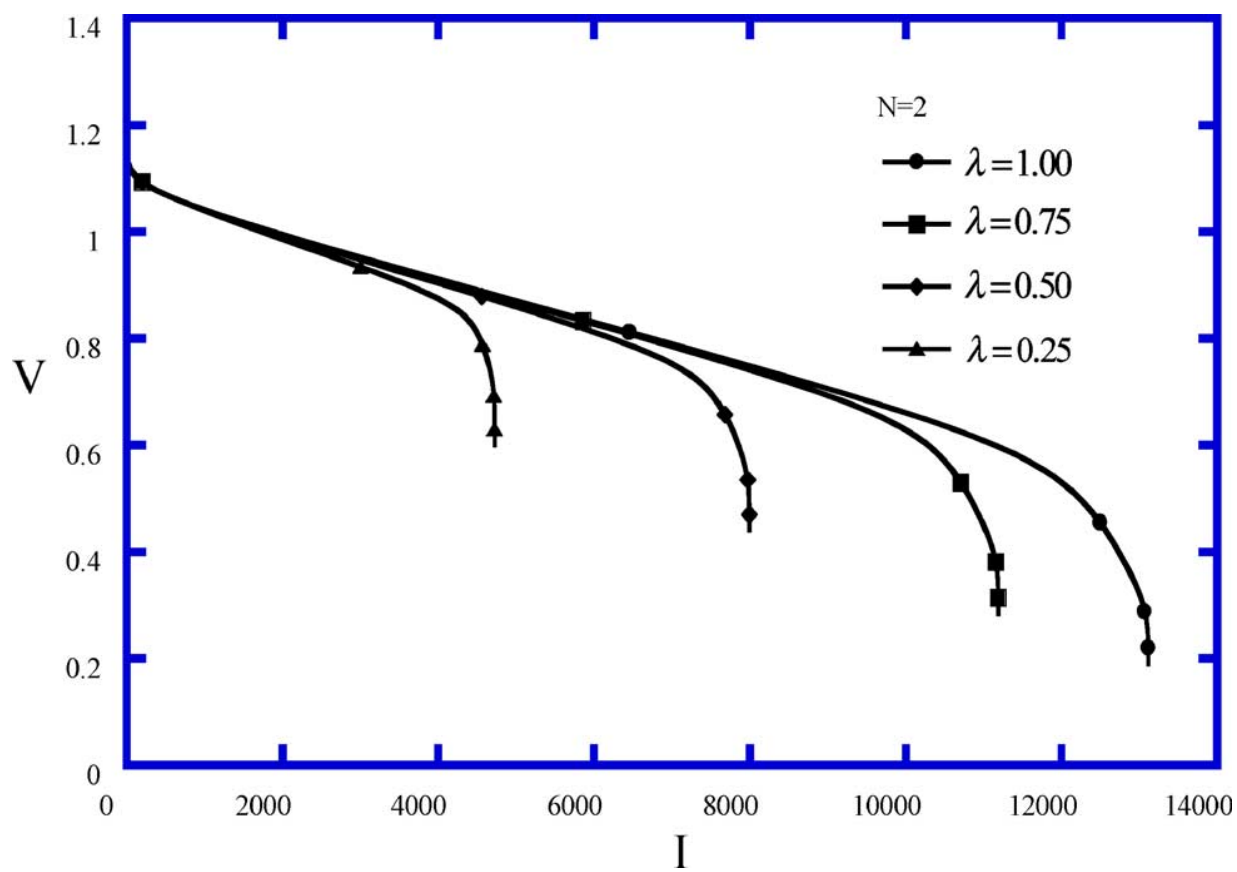

Fig. 7. Comparison of $I$ vs. $V$ performance for flow channel models with various channel width fractions. 

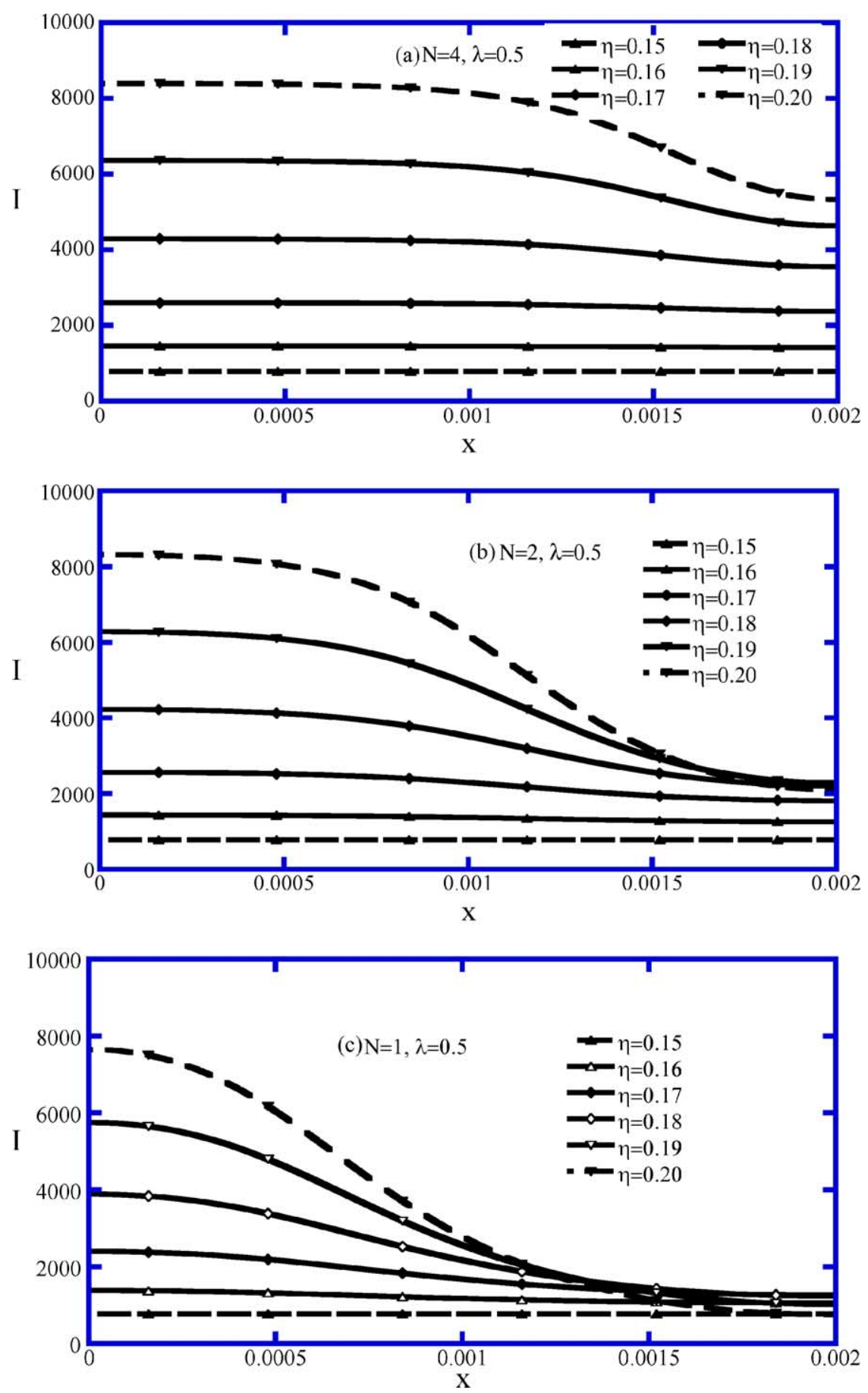

Fig. 8. Effect of surface overpotential on current density distributions along $x$-direction of models with $\lambda=0.5$ and channel number: (a) $N=4$; (b) $N=2$; (c) $N=1$.

gas in the across-the-cell direction does result, however, in a relatively non-uniformity in the $x$-distribution of the current density. Obviously, cell performance depends on the GDL porosity. In Fig. 12 the $I-V$ curves demonstrate that a better performance can be obtained by using a gas-diffusion layer of higher porosity. The remarkable drop in cell potential is caused by a mass transfer or concentration loss, which is a consequence of shortage of the reactant gas at high current density in which more reactant gas is required for fast reaction. A gas-diffusion layer of higher porosity has an ability of stronger diffusion transport, which is beneficial in that it supplements the reactant gas to the catalyst layer and thus shifts the occurrence of the performance drop to a higher value of the current density (Fig. 12). 


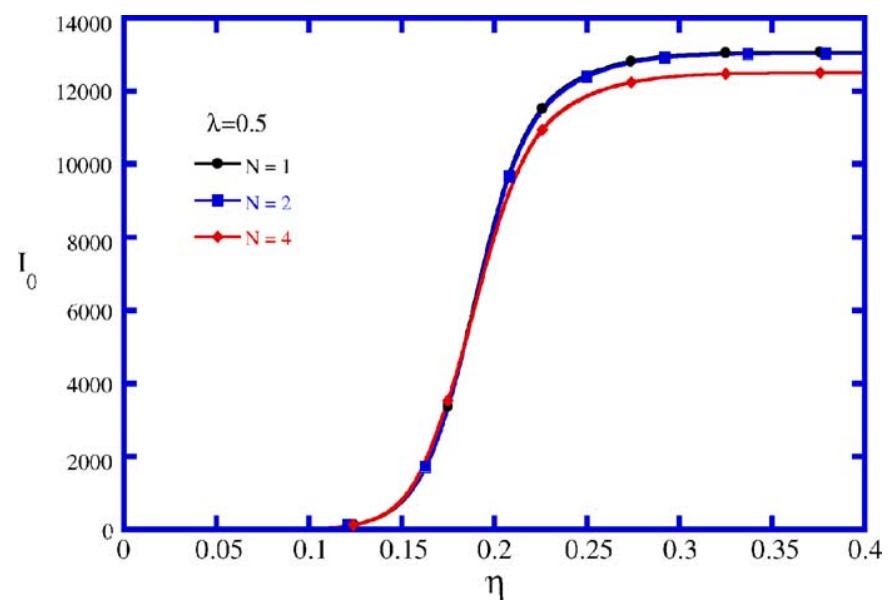

Fig. 9. Correlation of local maximum of current density and overpotential for channel models of $\lambda=0.5$ and $N=1,2$, and 4 .

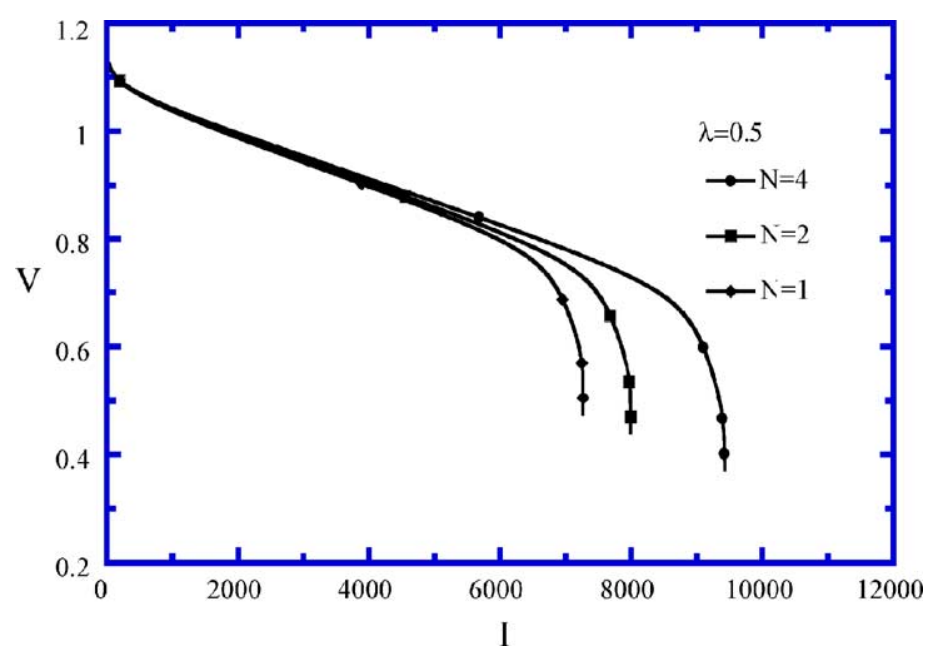

Fig. 10. $I$ vs. $V$ performance curves for flow channel models of $\lambda=0.5$ and $N=1,2$, and 4 .
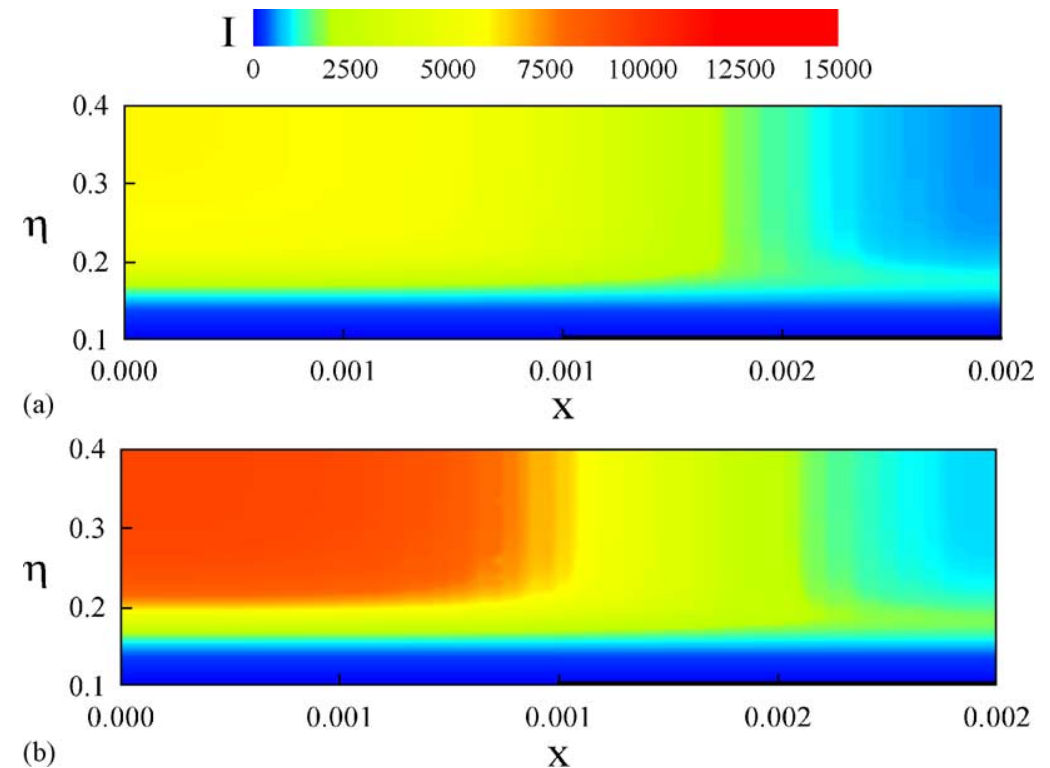

Fig. 11. Effects of gas-diffusion layer porosity on current density distributions along $x$-direction of models for $N=2, \lambda=0.5$ and various overpotential: (a) $\varepsilon_{1}=0.3$; (b) $\varepsilon_{1}=0.4$; (c) $\varepsilon_{1}=0.5$; (d) $\varepsilon_{1}=0.6$. 

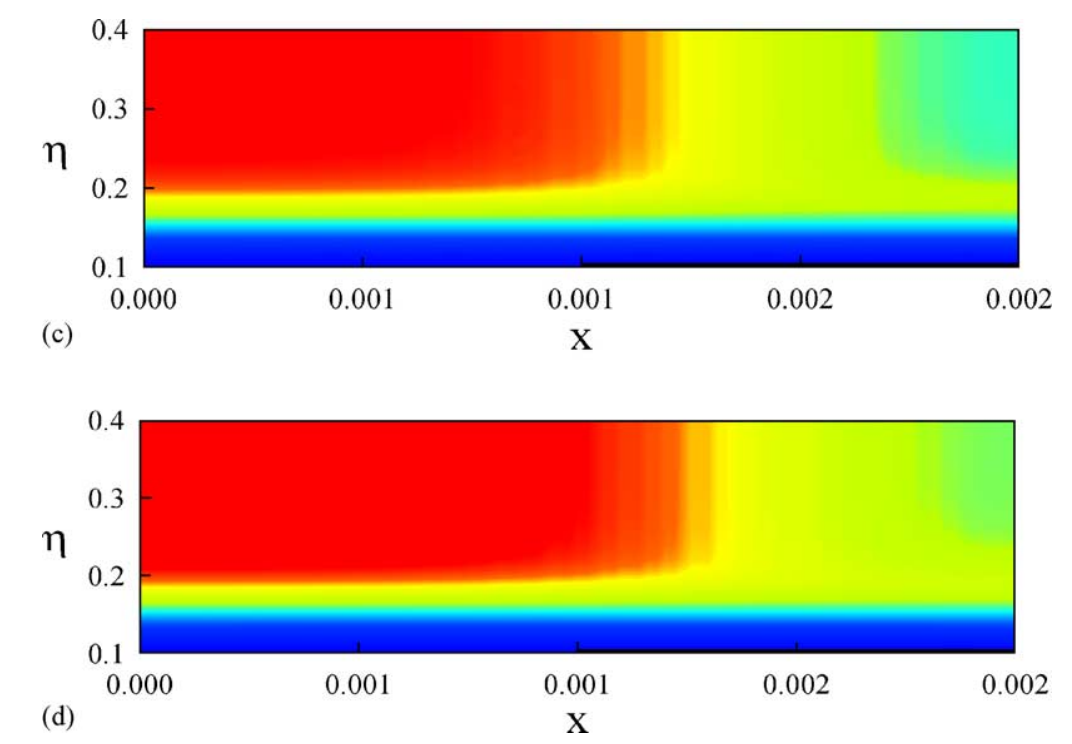

Fig. 11. (Continued).

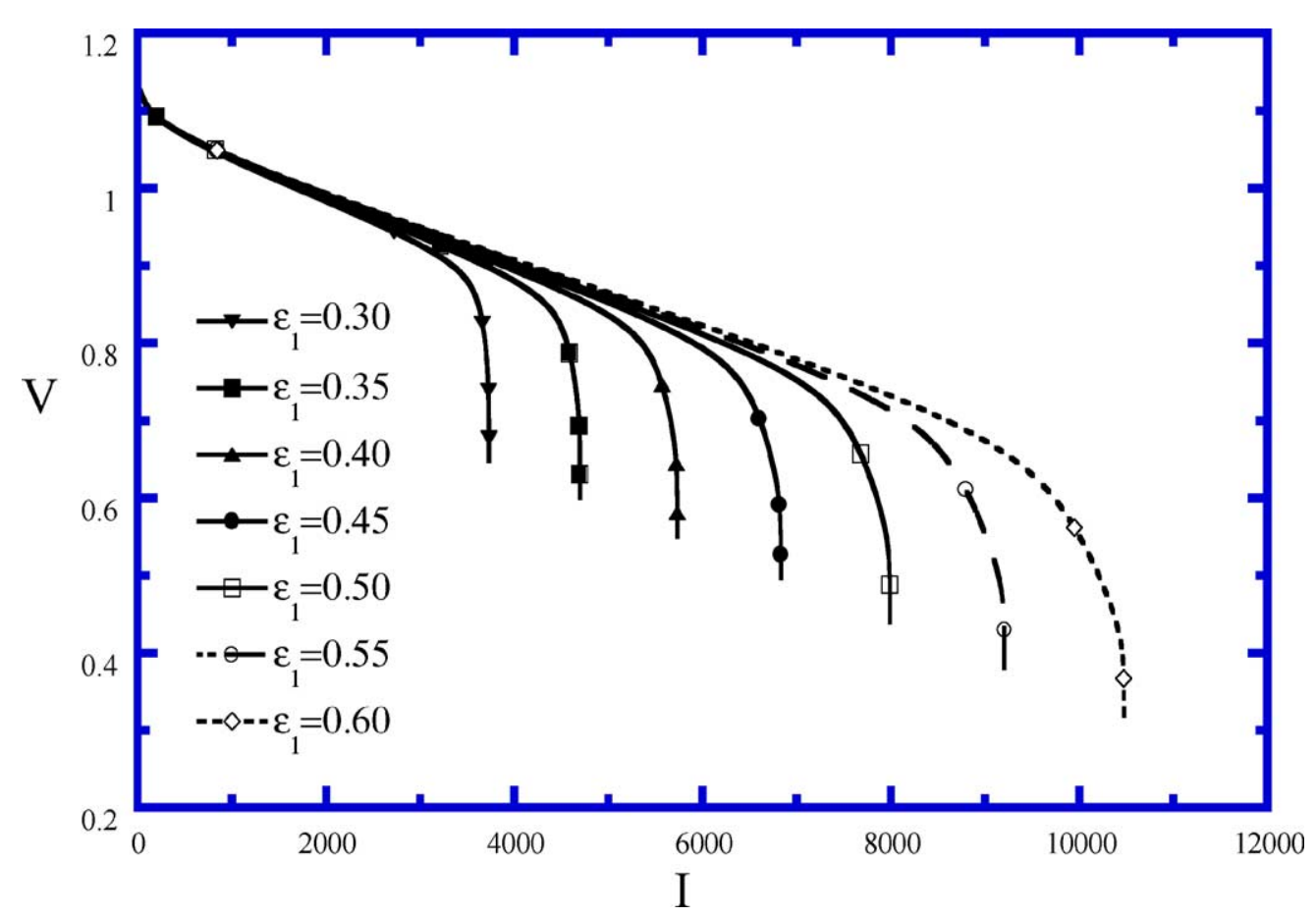

Fig. 12. Comparison of cell performance curves for models with porosity in range $0.3 \leq \varepsilon_{1} \leq 0.6$.

\subsection{Criterion for justification of one-dimensional model analysis}

From the current density results discussed above, it is revealed that uniform distribution of the current density can be attained at a low overpotential. To facilitate the analysis, a parameter is defined as the relative deviation of local current density along the $x$-direction $\left(I_{\max }-I_{\min }\right) / I_{\max }$, to judge of the uniformity of the current density distribution. $I_{\max }\left(=I_{0}\right)$ and $I_{\min }$ denote the maximum and minimum values. For $\left(I_{\max }-I_{\min }\right) / I_{\max } \leq 10 \%$, the current density dis- tribution can be regarded as a weak function of $x$. Therefore, the $x$-dependence can be neglected and an one-dimensional analysis can be regarded as appropriate; otherwise, at least two-dimensional analysis should be considered. At a higher overpotential, more reactant gas is required for the relatively strong and fast reaction, and therefore, the reactant gas from the flow channel may not have sufficient time to distribute uniformly over the width of the cell. With non-uniform distribution of the reactant mass fraction, the resultant current density distribution also has a noticeable non-uniformity. Based on the computations with the developed model, the 

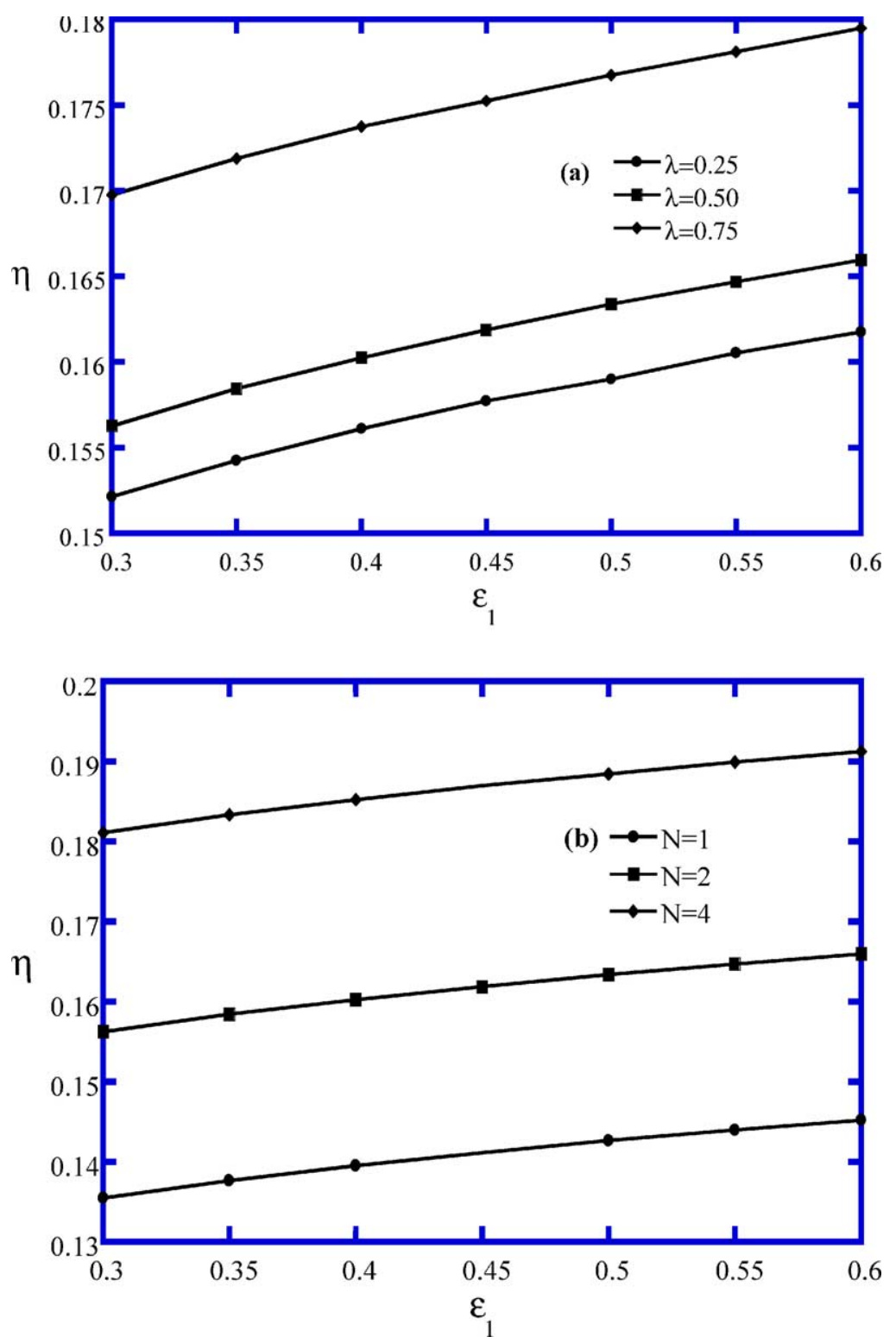

Fig. 13. Critical overpotentials for $10 \%$ deviation in uniformity of current density distribution.

values of overpotential for $\left(I_{\max }-I_{\min }\right) / I_{\max }=10 \%$ are plotted in Fig. 13. It is observed that the critical overpotential is increased with an increase in either $\lambda, N$, or $\varepsilon$. A correlation of $\eta_{\mathrm{cr}}=\eta_{\mathrm{cr}}(\lambda, N, \varepsilon)$ can be fitted with the present predictions. Finally, this gives:

$\eta_{\mathrm{cr}}=0.165 \lambda^{0.0935} N^{0.2033} \varepsilon^{0.0856}$

The calculated values of $\eta_{\text {cr }}$ by Eq. (12) with the parameters considered in the present analysis are of only less than $3 \%$ deviations from the numerical predictions. With a set of the given data of $\lambda, N$, and $\varepsilon$, the critical value, $\eta_{\mathrm{cr}}$, can be evaluated from Eq. (12). For a value of overpotential $\eta<\eta_{\mathrm{cr}}$, the one-dimensional analysis can be accepted for only $10 \%$ or less non-uniformity in prediction of the $x$-distribution on of the current density. This correlation may be employed as a guide for justification of the appropriateness of the one-dimensional approximation in the analysis.

\section{Concluding remarks}

A theoretical model has been developed to investigate the effects of flow distributor and diffusion layer morphology on the transport phenomena of reactant gas from flow channels to gas diffuser and the effects on performance of a PEMFC. By applying this two-dimensional mass transport model, the effects of overpotential, channel width fraction in the cross-sectional view of the anode flow distributor, number of flow channels, and porosity of the gas-diffusion layer/membrane on the resultant current density are 
investigated. The cell performance is evaluated by the calculated performance curves. Based on the results, the following conclusions can be drawn:

(i) Overpotential can affect significantly both the level and the uniformity of the local current density distribution. A low overpotential leads to a more uniform current density distribution. Based on the present results at various overpotentials, a correlation is proposed to serve as a criterion for justification of the validity of a simplified one-dimensional model.

(ii) With a fixed dimension of the flow distributor or bipolar plate, a larger channel width fraction results in a better cell performance. For a fixed channel width fraction, an increase in the number of flow-channel results in a more effective gas distribution, a more uniform local current density and, in turn, a better performance.

(iii) The morphology of the gas-diffusion layer in the present half-cell model is characterized by the porosity. With a porosity in the range $0.3-0.6$, a better cell performance can be obtained with higher porosity of the porous layer.

(iv) The present results provide a useful guide for the design of a flow distributor and a gas-diffusion layer via a better understanding of the effects of the parameters pertaining to the geometry and the morphology.
More profound investigations are needed to include the electrochemical effects for a more precise prediction of cell performance.

\section{Acknowledgements}

The study was supported by the National Science Council, the Republic of China through the grant numbers NSC-91-2218-E-211-001, NSC-91-2218-E-035-002, and NSC-92-2623-7-002-006-ET.

\section{References}

[1] A.C. West, T.F. Fuller, J. Appl. Electrochem. 6 (1996) 557-565.

[2] L.R. Jordan, A.K. Shukla, T. Behrsing, N.R. Avery, B.C. Muddle, M. Forsyth, J. Power Sources 86 (2000) 250-254.

[3] L.R. Jordan, A.K. Shukla, T. Behrsing, N.R. Avery, B.C. Muddle, M. Forsyth, J. Appl. Electrochem. 30 (2000) 641-646.

[4] V. Guran, R. Barbir, H. Liu, J. Electrochem. Soc. 147 (2000) 2468 2477.

[5] S. Dutta, S. Shimpalee, J.W. Van Zee, Int. J. Heat Mass Transfer 44 (2001) 2029-2042.

[6] E. Hontanon, M.J. Escudero, C. Bautista, P.L. Garcia-Ybarra, L. Daza, J. Power Sources 86 (2001) 363-368.

[7] R.J. Kee, P. Korada, K. Walters, M. Pavol, J. Power Sources 109 (2002) 148-159. 